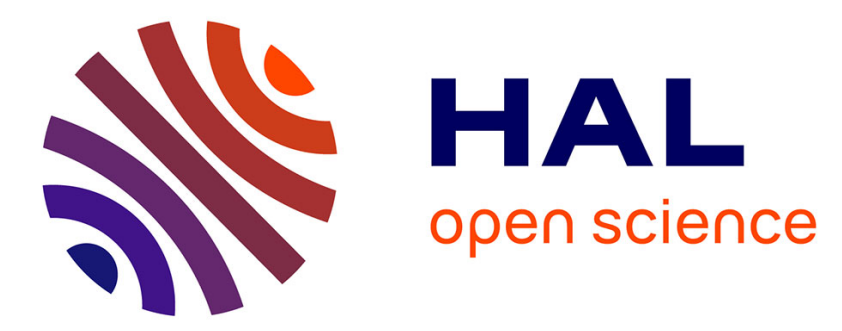

\title{
Sliding window proper orthogonal decomposition: Application to linear and nonlinear modal identification
}

Simon Clément, Sergio Bellizzi, Bruno Cochelin, Guillaume Ricciardi

\section{To cite this version:}

Simon Clément, Sergio Bellizzi, Bruno Cochelin, Guillaume Ricciardi. Sliding window proper orthogonal decomposition: Application to linear and nonlinear modal identification. Journal of Sound and Vibration, 2014, 333 (21), pp.5312-5323. 10.1016/j.jsv.2014.05.035 . hal-01038118

\section{HAL Id: hal-01038118 https://hal.science/hal-01038118}

Submitted on 29 Sep 2020

HAL is a multi-disciplinary open access archive for the deposit and dissemination of scientific research documents, whether they are published or not. The documents may come from teaching and research institutions in France or abroad, or from public or private research centers.
L'archive ouverte pluridisciplinaire HAL, est destinée au dépôt et à la diffusion de documents scientifiques de niveau recherche, publiés ou non, émanant des établissements d'enseignement et de recherche français ou étrangers, des laboratoires publics ou privés.

\section{(c)(1)}

Distributed under a Creative Commons Attribution| 4.0 International License 


\title{
Sliding Window Proper Orthogonal Decomposition : application to linear and nonlinear modal identification
}

\author{
Simon Clement*,**, Sergio Bellizzi**, Bruno Cochelin** and Guillaume Ricciardi* \\ *CEA Cadarache DEN/DTN/STCP/LHC, \\ Saint-Paul-Lez-Durance, 13108 Cedex, France \\ ${ }^{* *}$ LMA, CNRS, UPR 7051, Centrale Marseille, \\ Aix-Marseille Univ, Marseille Cedex 20, France
}

\begin{abstract}
The Proper Orthogonal Decomposition (POD) method is a tool well adapted to analyse vector signals whereas Continuous Gabor Transform (CGT) is suitable for scalar signal with multifrequency components. In this paper, a method named Sliding Window Proper Orthogonal Decomposition (SWPOD) combining POD and CGT to analyse Multi-Degrees-Of-Freedom (MDOF) vibration system responses is presented. SWPOD gives access to the evolution of the coherent spacial structures and their frequency components versus time. The method is of principal interest in the case of swept-sine excitation of linear or nonlinear systems to access the resonance frequencies, mode shapes and modal damping ratios. A procedure is proposed to extract the linear and non-linear normal modes of weakly damped MDOF mechanical systems and illustrated using numerical examples.
\end{abstract}

\section{Introduction}

The study of complex vibrating systems with numerous degrees of freedom containing nonlinearities is still a major challenge today. The well-known theory of linear modal analysis is limited in understanding the practical behavior of real systems, especially when nonlinearities have dominant effects on their behavior. A large variety of methods which are able to deal with nonlinearities have been proposed to characterize and/or identify systems. Time domain approaches, frequency domain approaches, time-frequency approaches, modal approaches have been considered. Moreover, the theory of nonlinear normal modes is promising, but not yet applicable to complex practical systems.

Time-frequency (or wavelet) analysis is a powerful signal processing tool to study signals with timedependent frequency content. It has been used to characterize and identify mechanical systems. For example, modal analysis of linear mechanical systems based on wavelet transform has been performed using output-only free time responses[1][2][3], and under ambient excitation[4]. These approaches are based on the notion of ridges and skeletons introduced for the continuous wavelet transform of scalar

Accepted Author Manuscript by Journal of Sound and Vibration 2014 
signals and differ with the choice of wavelet families. Variants from the use of skeleton can be found in [5], where a wavelet-based formula similar to the logarithmic decrement formula has been introduced to estimate damping. Some approaches have been extended to characterize nonlinear mechanical systems (see [2]). Specific methods have also been developed. In [6][7] for example, Continuous Gabor Transform (CGT), a time-frequency transform, is used to estimate the nonlinear normal modes of weakly damped nonlinear systems from free responses. Common to all these methods based on wavelet or time-frequency transform is that they are generally applied independently to each component of the response extracting the localized frequency content for each of them. It is a local method in the space domain.

On the other side, the Proper Orthogonal Decomposition (POD) method is a global approach characterizing spatial coherent structures. It has now emerged as a structural dynamics analysing tool for systems with a high number of Degrees Of Freedom (DOF). The physical interpretation of the Proper Orthogonal Modes (POMs) (the coherent structures) was investigated by several authors $[8,9,10,11]$ with the links between the POMs and the linear normal modes highlighted. In the case of lightly damped linear systems harmonically excited by an external force, it was shown [8] that a single POM captures all the energy and when the excitation frequency is near a resonance frequency, this POM is a linear normal mode of the system. However, in the case of a nonlinear system, the energy can be shared between several POMs and the results of a POD applied to the entire signal can be difficult to interpret as the physical characteristics of the system. For instance, the application of the POD to a nonlinear system excited at different discrete frequencies was investigated by Kerschen et al. [12], showing the appearance of a second energetic POM at frequencies where the nonlinearities of the structure are effective and a modal behavior depending strongly on the excitation frequency.

To analyse data involving complex evolution in time, frequency and space, it is thus relevant to combine POD and time-frequency transform. This combination is at the heart of the Sliding Window POD (SWPOD) method, which gives the evolution of the coherent structures of the data as well as their frequency components versus time. This ambivalent ability can be used to analyse complex simulated or experimental data, with the main structural and frequential characteristics of the signals extracted. The method can also be used to identify the resonance frequencies, mode shapes and damping ratios of a linear system, or to investigate the nonlinear normal modes (NNM) of a nonlinear system. Note that time-frequency transform and POD have already been combined in the context of noise reduction (see [13]), but the POD is applied to the time-frequency transform of a scalar signal and spacial dimension is not present. A method recently developed called Dynamic Mode Decomposition, when applied to experimental data, also gives a set of modes evolving with time, but with a different approach using the Koopman operator [14].

This paper is organized as follows. In Section 2, CGT and POD are first briefly recalled, followed by the description of the SWPOD method including numerical implementation. Section 3 is dedicated to the description of the nonlinear mechanical model used to show the potentiality of the method when applied to the responses obtained under linear swept sine excitation. Responses associated to low and high excitation levels are considered in Section 4 and section 5 respectively. In both cases, the mechanical interpretation of the results obtained with SWPOD is discussed, giving access to the modal parameters in linear (low excitation levels) and nonlinear cases (high excitation levels). 


\section{Presentation of the SWPOD method}

\subsection{The Continuous Gabor transform}

Continuous Gabor Transform (CGT) of a scalar signal $s(t)$ can be defined from the transform :

$$
G_{s}(\tau, f)=\int_{-\infty}^{+\infty} s(t) \overline{g(t-\tau)} \mathrm{e}^{-\mathrm{j} 2 \pi f(t-\tau)} \mathrm{d} t(\in \mathbb{C})
$$

where $g(t)$ is a scalar (real or complex) function named window function but also mother wavelet (this is an abuse of notation) and $\bar{g}$ denotes the complex conjugate of $g$. Several types of window functions can be used, such as Hamming, Hanning, Blackman windows, in which cases the signal is truncated and the Gabor transform is called Short Time Fourier Transform (STFT).

Gabor proposed to use Gaussian functions $\left(g(t)=\mathrm{e}^{-\frac{t^{2}}{2 \sigma^{2}}}\right.$ where $\left.\sigma>0\right)$ as window functions, which assure the most efficient utilization of the information as shown in [21]. In terms of windowing, $\sigma$ controls the effective width of the windowing. CGT extracts both time and frequency information from a signal, by applying windows sliding in time (realizing the time localization) before applying the Fourier transform. CGT can be used to capture the time evolution of the frequency content of the signal.

It is important to remember that the CGT can be inverted with the formula

$$
s(t)=\frac{1}{2 \pi\|g\|^{2}} \int_{-\infty}^{+\infty} \int_{-\infty}^{+\infty} G_{s}(\tau, f) g(t-\tau) \mathrm{e}^{\mathrm{j} 2 \pi f(t-\tau)} \mathrm{d} \tau \mathrm{d} f,
$$

where $\|g\|^{2}=\int_{-\infty}^{+\infty}|g(t)|^{2} \mathrm{~d} t$.

\subsection{The Proper Orthogonal Decomposition}

Let us consider a vector signal $\mathbf{S}(t)$ (with $N$ components) for $t \in \mathbb{R}$. Without loss of generality, we assume that all components have zero mean values, with respect to the mean operator (or time average, $\left.<.>=\int_{-\infty}^{+\infty}(.) \mathrm{d} t\right)$. The POD gives the most characteristic vectors $\boldsymbol{\Phi}$ representing the evolution of the vector signal $\mathbf{S}(t)$. These vectors can be found by maximizing the time average of the scalar product of the two vectors $\boldsymbol{\Phi}$ and $\mathbf{S}(t)$ :

$$
\text { Maximize }<\left|\mathbf{S}(t)^{T} \boldsymbol{\Phi}\right|^{2}>\text { with } \boldsymbol{\Phi}^{T} \boldsymbol{\Phi}=1 .
$$

The vectors which yield the maximum are solutions of the eigenvalue problem:

$$
\mathbf{R} \Phi=\lambda \boldsymbol{\Phi} \text { with } \mathbf{R}=<\mathbf{S}(t) \mathbf{S}(t)^{T}>.
$$

Let $\lambda_{1} \geq \lambda_{2} \cdots \geq \lambda_{N}$ be the ordered eigenvalues, then the associated $N$ orthonormal vectors $\boldsymbol{\Phi}_{i}$ can be used as a basis to expand $\mathbf{S}(t)$ as

$$
\mathbf{S}(t)=\sum_{i=1}^{N} \boldsymbol{\Phi}_{i} \sqrt{\lambda_{i}} \eta_{i}(t),
$$

where the scalar signals $\eta_{i}(t)$ are defined as $\eta_{i}(t)=\boldsymbol{\Phi}_{i}^{T} \mathbf{S}(t) / \sqrt{\lambda_{i}}$ and satisfy the orthogonal property $<\eta_{i}(t) \eta_{j}(t)>=\delta_{i j}$ (where $\delta_{i j}$ is the Kronecker delta). The vectors $\boldsymbol{\Phi}_{i}$ are the POMs giving the 
coherent structures. The $\eta_{i}(t)$ called Proper Orthogonal Components (POCs) are their normalized time evolutions representing the dynamics of the signal. The reals $\lambda_{i}$ are the Proper Orthogonal Values (POVs) defining the energy captured by each POM.

Among the numerous applications of the POD stands reduced order modelling, which uses its ability to reduce the number of POMs necessary to represent the signal from $N$ to $N^{r}$ if the following condition is satisfied:

$$
\sum_{i=1}^{N^{r}} \lambda_{i}>99 \% \sum_{i=1}^{N} \lambda_{i}
$$

This characteristic of the POD allows analysing complex data with a high number of components and in the case of structural dynamics, systems with numerous degrees of freedom.

Finally, as mentioned in the introduction, it was shown in [8] that for a lightly damped system harmonically excited, only one POM captures all the energy of the system. Observing the sharing of energy between POMs can thus be a way to detect the presence of high damping or nonlinearities for harmonically excited systems.

\subsection{The proposed method}

In order to analyse a vector signal $\mathbf{S}(t)$ and capture its spatial evolution as well as its frequency content, POD and CGT can be combined, giving the Sliding Window POD (SWPOD) method.

The window function is first applied to the vector signal $\mathbf{S}(t)$ giving the windowed vector signal

$$
\mathbf{S}^{\tau}(t)=\mathbf{S}(t) g(t-\tau)
$$

Next, the windowed vector signal is expanded using the POD as

$$
\mathbf{S}^{\tau}(t)=\sum_{i=1}^{N} \boldsymbol{\Phi}_{i}^{\tau} \sqrt{\lambda_{i}^{\tau}} \eta_{i}^{\tau}(t) .
$$

Note that it is possible to reduce the number of terms in the expansion from $N$ to $N^{\tau}$, with a criterion of the type $\sum_{i=1}^{N^{\tau}} \lambda_{i}^{\tau} \geq 0.99 \sum_{i=1}^{N} \lambda_{i}^{\tau}$. Finally the Gabor transform of $\mathbf{S}(t)$ results in the sliding window POD form

$$
\begin{aligned}
\mathbf{G}_{\mathbf{S}}(\tau, f) & =\int_{-\infty}^{+\infty} \sum_{i=1}^{N} \boldsymbol{\Phi}_{i}^{\tau} \sqrt{\lambda_{i}^{\tau}} \eta_{i}^{\tau}(t) \mathrm{e}^{-\mathrm{j} 2 \pi f(t-\tau)} \mathrm{d} t \\
& =\sum_{i=1}^{N} \boldsymbol{\Phi}_{i}^{\tau} \sqrt{\lambda_{i}^{\tau}} \tilde{G}_{i}(\tau, f)
\end{aligned}
$$

where $\tilde{G}_{i}(\tau, f)=\int_{-\infty}^{+\infty} \eta_{i}^{\tau}(t) \mathrm{e}^{-\mathrm{j} 2 \pi f(t-\tau)} \mathrm{d} t$.

For each time $\tau$, the sliding window POD form (9) gives access for $i=1, \cdots, N$ to

- the localized POMs, $\boldsymbol{\Phi}_{i}^{\tau}$, which characterize the coherent structures associated to the vector signal localized around the time $\tau$;

- the localized POVs, $\lambda_{i}^{\tau}$, which characterize the repartition of energy between the localized POMs; 
- the localized POCs, $\eta_{i}^{\tau}(t)$, and their frequency content, $\tilde{G}_{i}(\tau, f)$.

As well as for the Gabor transform, an inverse formula to (9) is also valid and reads as

$$
\mathbf{S}(t)=\frac{1}{2 \pi\|g\|^{2}} \sum_{i=1}^{N} \int_{-\infty}^{+\infty} \boldsymbol{\Phi}_{i}^{\tau} \sqrt{\lambda_{i}^{\tau}} \int_{-\infty}^{+\infty} \hat{G}_{i}(\tau, f) g(t-\tau) \mathrm{e}^{\mathrm{j} 2 \pi f(t-\tau)} \mathrm{d} f \mathrm{~d} \tau .
$$

\subsection{Practical application of the SWPOD}

We will assume in what follows that the vector signal $\mathbf{S}(t)$ is sampled at the sampling frequency $f_{s}$ (respecting the Nyquist theorem) and acquired on $[0, T]$ at $P$ sampled times $t_{p}=p / f_{s}$ for $p=1, \cdots, P$ and $T=t_{P}$. We will also assume that $P$ is a power of two (for the optimization of the Fast Fourier Transform (FFT)).

A $N \times P$ matrix $\mathcal{S}$ is thus formed, in which the terms $S_{n}\left(t_{p}\right)$ give the component $n$ of $\mathbf{S}(t)$ at the time $t_{p}$ as

$$
\mathcal{S}=\left[\begin{array}{cccc}
S_{1}\left(t_{1}\right) & S_{1}\left(t_{2}\right) & \ldots & S_{1}\left(t_{P}\right) \\
S_{2}\left(t_{1}\right) & S_{2}\left(t_{2}\right) & \ldots & S_{2}\left(t_{P}\right) \\
\vdots & \vdots & \ddots & \vdots \\
S_{N}\left(t_{1}\right) & S_{N}\left(t_{2}\right) & \ldots & S_{N}\left(t_{P}\right)
\end{array}\right]
$$

The window function $g(t)$ is sampled with the same sampling frequency and, for each time $t_{p}$, a $N \times P$ matrix $\mathcal{W}^{p}$ is formed as

$$
\mathcal{W}^{p}=\left[\begin{array}{ccccccc}
g\left(t_{1}-t_{p}\right) & \ldots & g\left(-t_{1}\right) & g(0) & g\left(t_{1}\right) & \ldots & g\left(t_{P}-t_{p}\right) \\
\vdots & \vdots & \vdots & \vdots & \vdots & \vdots & \vdots \\
g\left(t_{1}-t_{p}\right) & \ldots & g\left(-t_{1}\right) & g(0) & g\left(t_{1}\right) & \ldots & g\left(t_{P}-t_{p}\right)
\end{array}\right] .
$$

The windowed vector signal around the time $t_{p}$ is obtained as

$$
\mathcal{S}^{p}=\mathcal{S} \circ \mathcal{W}^{p}
$$

where o denotes the Hadamard (or term to term) product. The POD can then be performed directly through a singular value decomposition, as shown in [8], leading to the matrix decomposition :

$$
\mathcal{S}^{p}=\mathcal{U}^{p} \boldsymbol{\Sigma}^{p} \mathcal{V}^{p^{T}}
$$

where $\mathcal{U}^{p}$ and $\mathcal{V}^{p}$ are orthogonal and $\boldsymbol{\Sigma}^{p}$ is diagonal. The columns of $\mathcal{U}^{p}$ are the localized POMs $\boldsymbol{\Phi}_{i}^{p}$. The diagonal terms of $\boldsymbol{\Sigma}^{p}$ are the square roots of the localized POVs, $\sigma_{i}^{p}=\sqrt{\lambda_{i}^{p}}$. The columns of $\mathcal{V}^{p}$ are the sampled localized normalized POCs, $\eta_{i}^{p}(t)$. The frequency content $\tilde{G}_{i}\left(t_{p}, f\right)$ is deduced from the FFT of $\eta_{i}^{p}(t)$ at the discrete frequencies $f_{k}=k f_{s} / P$.

The evolution of the most energetic localized POM with respect to time $t_{p}$ is characterized in terms of energy by the time-energy plot $\left(t_{p}, \lambda_{1}^{p}\right)$, in terms of coherent structure by the time-space plot $\left(t_{p}, \boldsymbol{\Phi}_{1}^{p}\right)$ and in terms of frequency content by the time-frequency plot $\left(t_{p}, \tilde{G}_{1}\left(t_{p}, f_{k}\right)\right)$. The second most energetic localized POM is characterized by $\left(t_{p}, \lambda_{2}^{p}\right),\left(t_{p}, \boldsymbol{\Phi}_{2}^{p}\right)$ and $\left(t_{p}, \tilde{G}_{2}\left(t_{p}, f_{k}\right)\right)$. This analysis can be 
performed for all the localized modes contributing to the dynamics of the signal and selected with a criterion of the type $\sum_{i=1}^{N^{p}} \lambda_{i}^{p} \geq 0.99 \sum_{i=1}^{N} \lambda_{i}^{p}$.

Although the temporal length of the window function as represented by Eq. (12) is $T$, its effective length $T_{e}$ depends on the bandwidth of the window $g(t)$ and contains a number of samples $P_{e}=T_{e} f_{s}$. $T_{e}$ has an influence on the resolution of the analysis in time and frequency and the choice of $T_{e}$ depends on the properties of the signal to analyse (changes in dynamic and frequency content). Consider a signal containing temporal events at least distant from $T_{\text {event }}$ and characteristic frequencies separated by a minimum of $F_{\text {event }}$. $T_{e}$ has to be short enough to separate the temporal events, giving the condition $T_{e}<T_{\text {event }}$. Moreover, the frequency resolution of the analysis is given by $\delta f=f_{s} / P_{e}=1 / T_{e}$ and has to be inferior to $F_{\text {event }}$, leading to the second condition $T_{e}>1 / F_{\text {event }}$. When gaussian window functions are used, $T_{e}$ can be estimated as $T_{e}=6 \sigma$.

Finally, in practical applications, the SWPOD method can be performed at a reduced number $P_{r}$ of time steps instead of $P$, giving the localized parameters $\lambda_{i}^{\tau}, \Phi_{i}^{\tau}$ and $\eta_{i}^{\tau}(t)$ at $P_{r}$ regularly spread times $\tau$.

\section{Mechanical system under study}

The system considered in this paper is shown in Fig. 1. It is a 3-DOF spring-mass oscillator with linear springs and dashpots (light damping) having both end points related to the ground. The central mass is also connected to the ground with a cubic spring. The system is excited by applying a force on the first mass.

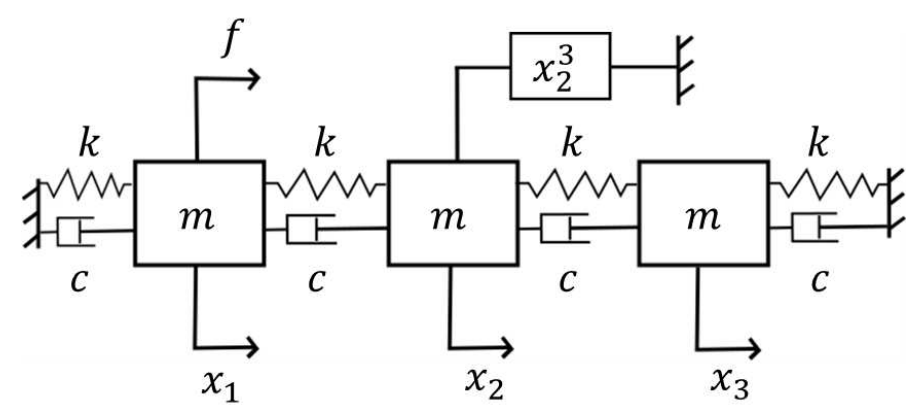

Figure 1: The nonlinear studied system.

The equations of motion reads as :

$$
\mathbf{M} \ddot{\mathbf{X}}+\mathbf{C} \dot{\mathbf{X}}+\mathbf{K X}+g(\mathbf{X}) \mathbf{V}=f(t) \mathbf{B}
$$

with $\mathbf{X}=\left(x_{1}, x_{2}, x_{3}\right)^{T}, \mathbf{V}=(0,1,0)^{T}, g(\mathbf{X})=x_{2}^{3}, \mathbf{B}=(1,0,0)^{T}$ and the following mass and stiffness matrices

$$
\mathbf{M}=\left(\begin{array}{ccc}
m & 0 & 0 \\
0 & m & 0 \\
0 & 0 & m
\end{array}\right), \mathbf{K}=\left(\begin{array}{ccc}
2 k & -k & 0 \\
-k & 2 k & -k \\
0 & -k & 2 k
\end{array}\right)
$$

The resonance frequencies of the underlying linear system are given by $\omega_{1}=\sqrt{(2-\sqrt{2}) k / m}, \omega_{2}=$ $\sqrt{2 k / m}$ and $\omega_{3}=\sqrt{(2+\sqrt{2}) k / m}$ with their corresponding linear normal modes $\Psi_{1}=(1 / \sqrt{2}, 1,1 / \sqrt{2})^{T}$, 
$\boldsymbol{\Psi}_{2}=(1,0,-1)^{T}$ and $\boldsymbol{\Psi}_{3}=(-1 / \sqrt{2}, 1,-1 / \sqrt{2})^{T}$. Proportional damping is considered by taking $\mathbf{C}=\frac{2 \xi_{1}}{\omega_{1}} \mathbf{K}$, with $\xi_{1}(>0)$ fixing the damping ratio of the first linear mode.

Considering the modal matrix $\boldsymbol{\Psi}=\left[\boldsymbol{\Psi}_{1} \boldsymbol{\Psi}_{2} \boldsymbol{\Psi}_{3}\right]$, the equations of motion can be reduced to :

$$
\mathbf{M}_{\Psi} \ddot{\mathbf{Q}}+\mathbf{C}_{\boldsymbol{\Psi}} \dot{\mathbf{Q}}+\mathbf{K}_{\Psi} \mathbf{Q}+g(\boldsymbol{\Psi} \mathbf{Q}) \Psi^{T} \mathbf{V}=f(t) \Psi^{T} \mathbf{B}
$$

with $\mathbf{Q}=\left(q_{1}, q_{2}, q_{3}\right)^{T}, \mathbf{X}=\mathbf{\Psi} \mathbf{Q}, \mathbf{C}_{\Psi}=\frac{2 \xi_{1}}{\omega_{1}} \mathbf{K}_{\Psi}$ and

$$
\mathbf{M}_{\boldsymbol{\Psi}}=\left(\begin{array}{ccc}
2 m & 0 & 0 \\
0 & 2 m & 0 \\
0 & 0 & 2 m
\end{array}\right), \mathbf{K}_{\boldsymbol{\Psi}}=\left(\begin{array}{ccc}
2(2-\sqrt{2}) k & 0 & 0 \\
0 & 4 k & 0 \\
0 & 0 & 2(2+\sqrt{2}) k
\end{array}\right)
$$

Assuming that $m=1 \mathrm{~kg}$ and $k=500 \mathrm{~m} / \mathrm{N}$, the natural frequencies of the underlying linear system are $f_{1}^{0}=2.72 \mathrm{~Hz}, f_{2}^{0}=5.03 \mathrm{~Hz}$ and $f_{3}^{0}=6.58 \mathrm{~Hz}$. At low excitation level, characteristic frequencies are separated by a minimum of $F_{\text {event }}=1.5 \mathrm{~Hz}$.

In this study, a linear swept sine is used for the excitation $f(t)$ under the form :

$$
f(t)=F \sin (\varphi(t)) \quad \text { with } \quad \varphi(t)=\frac{1}{2} \frac{2 \pi\left(f_{\max }-f_{\min }\right)}{T_{\text {sweep }}} t^{2}+2 \pi f_{\min } t+\varphi_{0},
$$

where $F$ is a constant amplitude, $T_{\text {sweep }}$ the time to go from the minimum frequency $f_{\min }$ to the maximum $f_{\max }$ and $\varphi_{0}$ the phase at the origin, which is chosen equal to zero. More information on systems excited by swept sines can be found in references [19] and [20].

In our case, we will perform tests with an excitation frequency varying from $f_{\min }=0.05 \mathrm{~Hz}$ to $f_{\max }=10 . \mathrm{Hz}$ over $T_{\text {sweep }}=400 \mathrm{~s}$. At low excitation level, temporal events are at least separated by

$$
T_{\text {event }}=\frac{F_{\text {event }} T_{\text {sweep }}}{f_{\text {max }}-f_{\text {min }}} \approx 30 \mathrm{~s} .
$$

The equations of motion (15) have been solved numerically using the Newmark method with a sampling frequency $f_{s}=200 \mathrm{~Hz}$ and zero initial conditions. The signal is produced on $[0, T]$ with $T=T_{\text {sweep }}$ corresponding to $P=T_{\text {sweep }} f_{s}$ sampled times. Then, the SWPOD method is applied to the vector signal $\mathbf{S}(t)=\left(x_{1}(t), x_{2}(t), x_{3}(t)\right)^{T}$ which characterizes the time evolution of the displacements of the three masses.

Finally, the effective length of the windows $T_{e}$ is defined with $\sigma=20 / 6$ corresponding to $T_{e} \approx 20$ s satisfying $T_{e}>1 / F_{\text {event }}$ and $T_{e}<T_{\text {event }}$.

In the following two sections, the results obtained with SWPOD method at respectively low and high excitation levels are discussed.

\section{Low excitation levels (linear behavior)}

Two damping ratios are considered in this section, with the excitation level $F=10 \mathrm{~N}$. The behavior of the system is expected to be linear. 


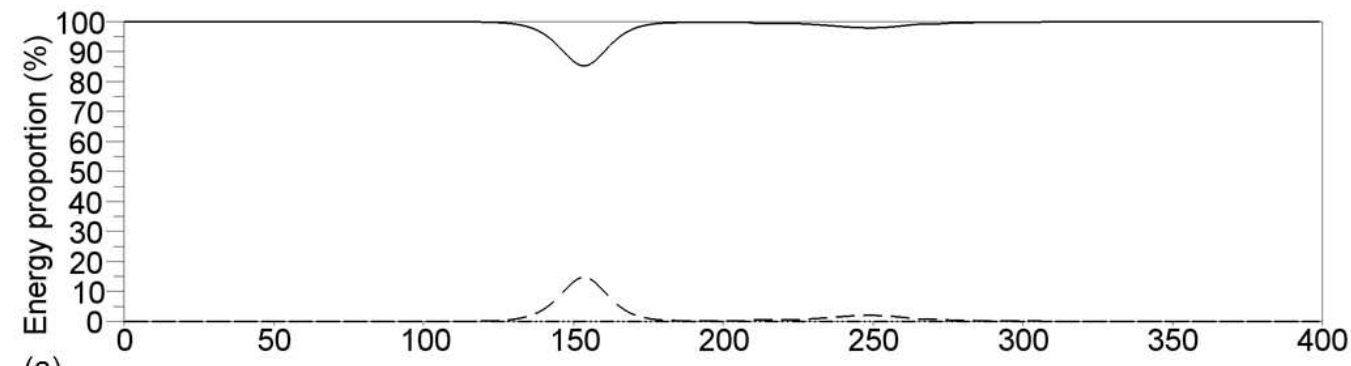

(a)
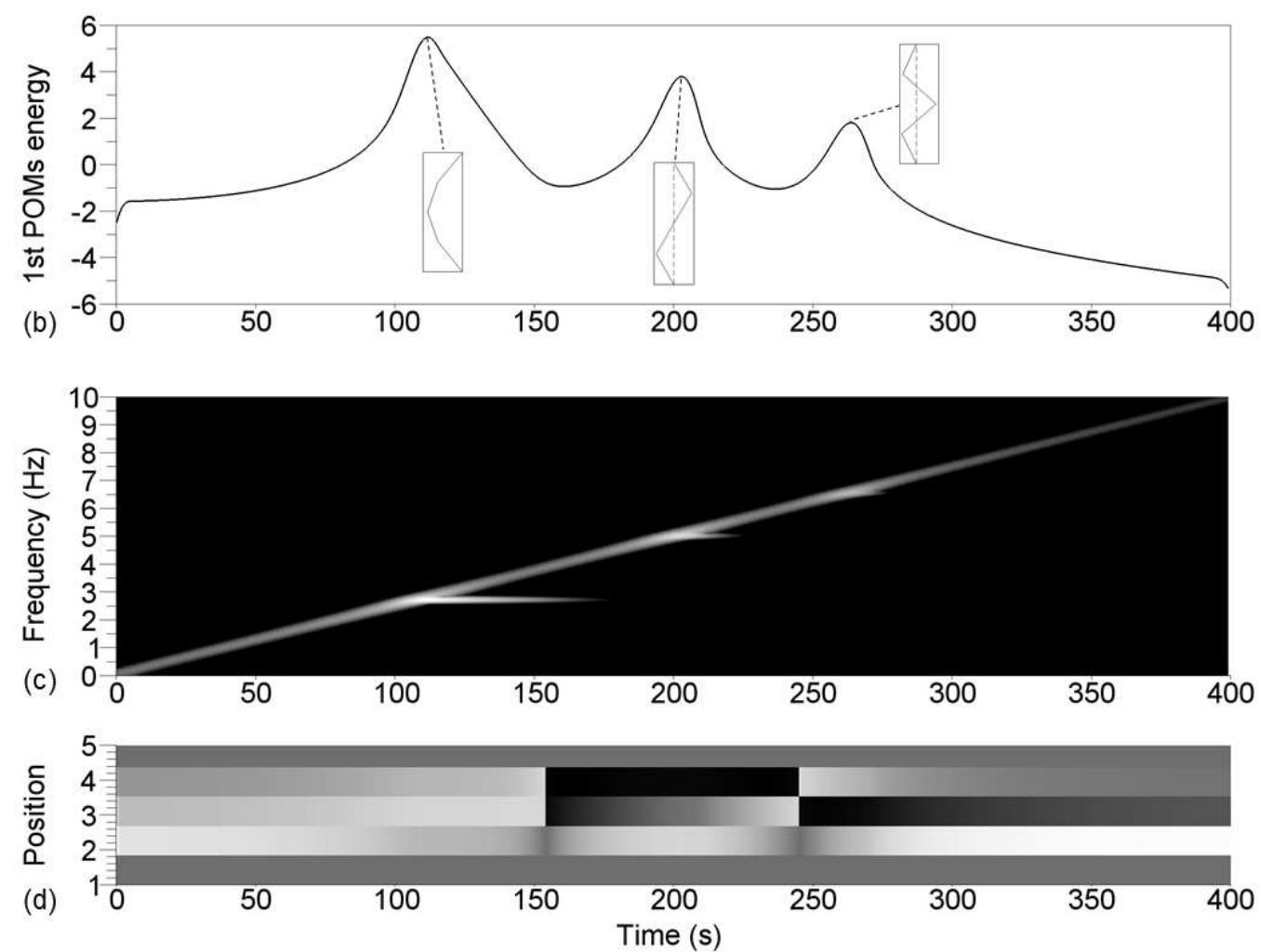

Figure 2: SWPOD analysis of a test with $\xi_{1}=0.5 \%$ and $F=10$ N. (a) Proportion of energy captured by the first 3 POMs $\lambda_{1}^{\tau} / \sum_{i=1}^{3} \lambda_{i}^{\tau}$ (solid line), $\lambda_{2}^{\tau} / \sum_{i=1}^{3} \lambda_{i}^{\tau}$ (dashed) and $\lambda_{1}^{\tau} / \sum_{i=1}^{3} \lambda_{i}^{\tau}$ (dotted). (b) Logarithmic representation of the energy captured by the first POM $\ln \left(\lambda_{1}^{\tau}\right)$. (c) Time-frequency representation of the first POCs multiplied by the POVs $\lambda_{1}^{\tau} \hat{G}_{1}(\tau, f)$. (d) Time evolution of the first POM with its two extremities: white (black) for high (low) displacements, the extremities giving the color associated to a zero value.

\subsection{Case with moderate damping}

The first test was carried out with the damping ratio $\xi_{1}=0.5 \%$ on the first mode of the underlying linear system. Fig. 2 shows the results obtained with the SWPOD. The analysis was performed at $P_{r}=400$ time steps.

The proportions of energy captured by the first three localized POMs are given in Fig. 2(a), showing that the first localized POM is largely dominant and captures more than $99 \%$ of the total energy during 
most of the test. However, a strong energy sharing between the first two POMs can be observed around $150 \mathrm{~s}$.

The localized POV $\lambda_{1}^{\tau}$, which characterizes the energy captured by the first localized POM, is given in Fig. 2(b) (time-energy plot) in logarithmic scale. Three local maxima appear at $\tau_{1}=112 \mathrm{~s}, \tau_{2}=203$ $\mathrm{s}$ and $\tau_{3}=264 \mathrm{~s}$ for which the associated localized POM $\boldsymbol{\Phi}_{1}^{\tau_{i}}$ are shown. These shapes are very close to the normal modes of the underlying linear system.

The product $\lambda_{1}^{\tau} \tilde{G}_{1}(\tau, f)$ is shown in a time-frequency plot in Fig. 2(c), characterizing the frequency evolution of the first localized POM. The main frequency content (the oblique line) of the signal follows the linear sweep, with small horizontal lines around $2.73 \mathrm{~Hz}$ after $110 \mathrm{~s}, 5.04 \mathrm{~Hz}$ after $200 \mathrm{~s}$ and 6.57 $\mathrm{Hz}$ after $260 \mathrm{~s}$.

Finally, the time evolution of the shape of the first localized POM (the time-space plot) is given in Fig. 2(d), showing that the system vibrates successively in accordance with the first, second and third normal modes of the underlying linear system.

\subsection{Case with low damping}

In order to bring a better understanding of the results obtained above and show the ability of the method to deal with complex data, a second test was carried out, reducing the damping ratio $\xi_{1}$ from $0.5 \%$ to $0.1 \%$. The results are reported in Fig. 3. Considering the high sharing of energy between localized POMs and the higher complexity of the behavior of the system, it is interesting to observe the evolution of the second and third localized POMs, reported in Fig. 4 in terms of time-frequency plot and time-space plot.

As shown in Fig. 3(a), the reduction of the damping strongly modifies the sharing of energy between localized POMs. The first energy sharing previously observed with $\xi_{1}=0.5 \%$ at $\tau=150$ s disappeared and more complex energy sharings appear. In Fig. 3(b), the local maxima of energy are detected at the same times $\tau_{1}=112 \mathrm{~s}, \tau_{2}=203 \mathrm{~s}$ and $\tau_{3}=264 \mathrm{~s}$, for which the associated localized POMs are again very close to the linear normal modes of the system.

The time-frequency plot (see Fig. 3(c)) shows that the horizontal frequency lines, which are initiated passing the resonance frequencies of the underlying linear system, persist on larger time intervals. At the times where two horizontal frequency lines are present (between $\tau=200 \mathrm{~s}$ and $\tau=300 \mathrm{~s}$ ), the energy captured by the first POM is localized around one of these frequencies, alternating between the frequency lines.

The time-frequency plots of the second and third localized POMs are given in Fig. 4(a,c). The energy is localized in time intervals where energy sharings are observed (Fig. 3(a)). The time-frequency plots have the same structure as the time-frequency plot of the first localized POM, with the oblique frequency line corresponding to the linear sweep component and the horizontal frequency lines initiated at the resonance frequencies of the underlying linear system. Alternances between the horizontal frequency lines (and the oblique line) can also be observed, opposite to the ones of the first POM.

During the time intervals where horizontal frequency lines persist, the time-energy plot (see Fig. 3(b)) shows a linear decrease of energy, with a change of slope when the previous alternances appear. These slopes are strongly linked to the modal parameters of the system and will be used in the next section to identify the damping of the linear normal modes.

The time-space plots (Fig. 3(d) and Fig. 4(b,d)) show that the contribution of the horizontal frequency lines to the vibration of the system are in accordance with the associated normal modes of 


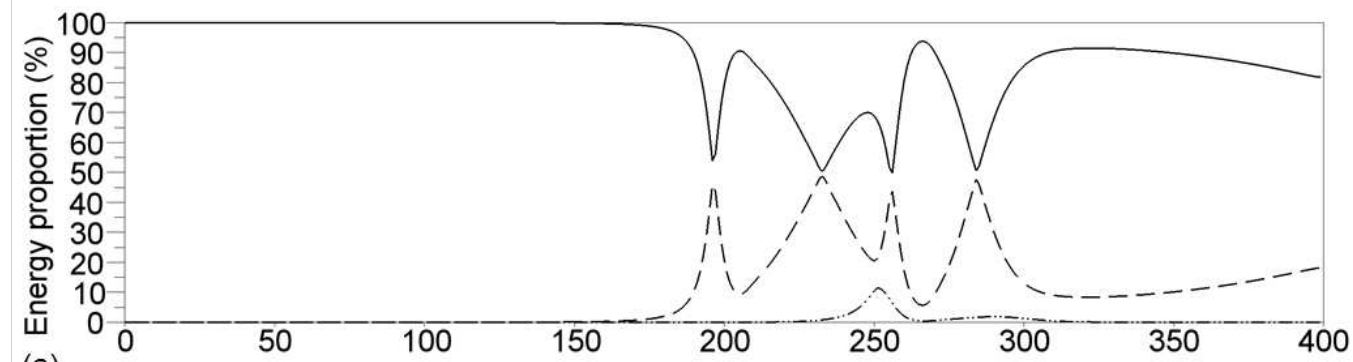

(a)
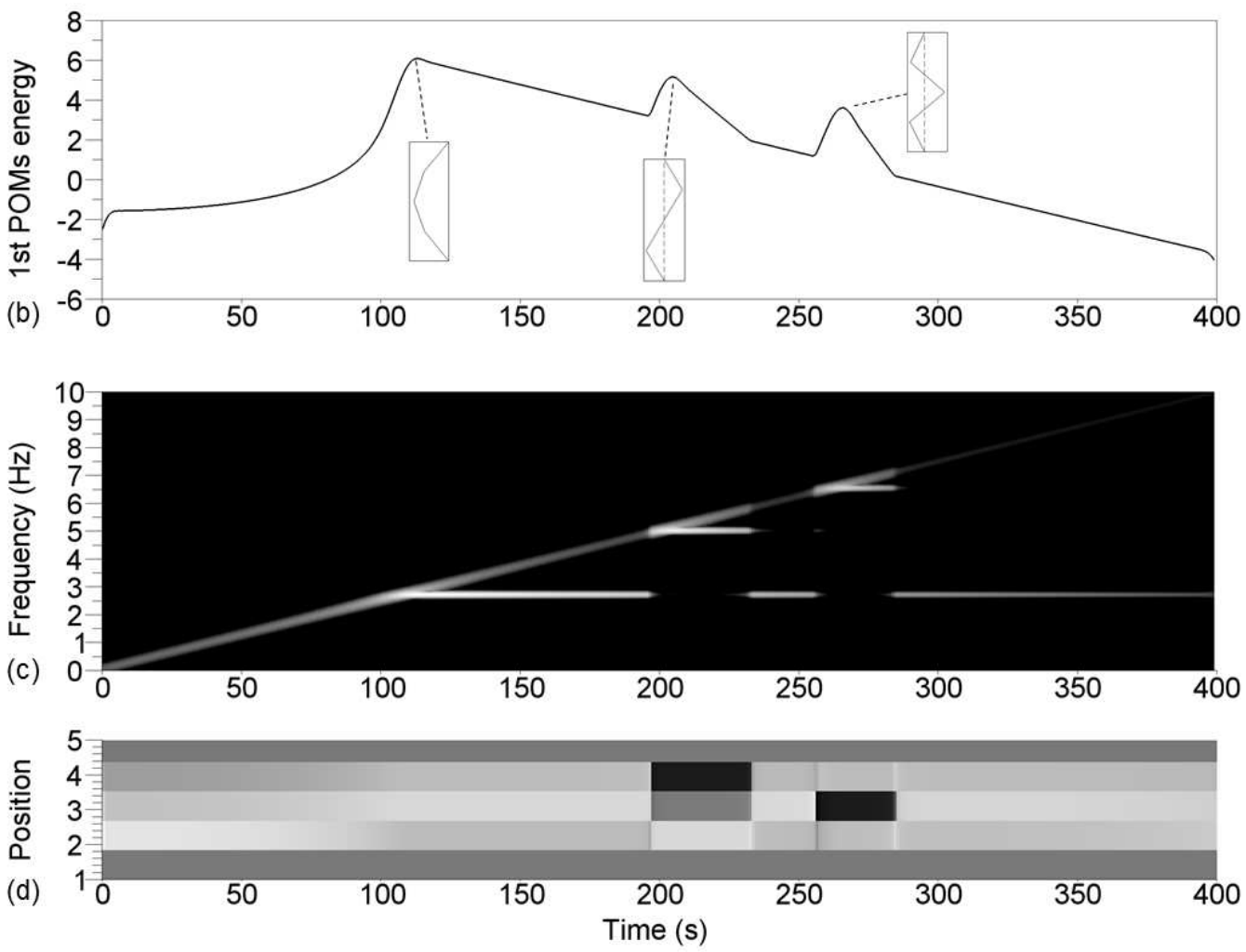

Figure 3: Results for a test with $\xi_{1}=0.1 \%$ and $F=10$ N. (a) Proportion of energy captured by the first (solid line), second (dashed) and third (dotted) POMs. (b) Energy captured by the first POM. (c) Time-frequency representation of the first POC multiplied by the POV $\lambda_{1}^{\tau} \hat{G}_{1}(\tau, f)$. (d) Time evolution of the first $\mathrm{POM} \Phi_{1}^{\tau}$.

the underlying linear system.

Global observations on the results were given above. In the next section, an in-depth analysis of the results will be presented, with the interpretation of the energy sharing detailed and the identification of the modal dampings, frequencies and mode shapes. 

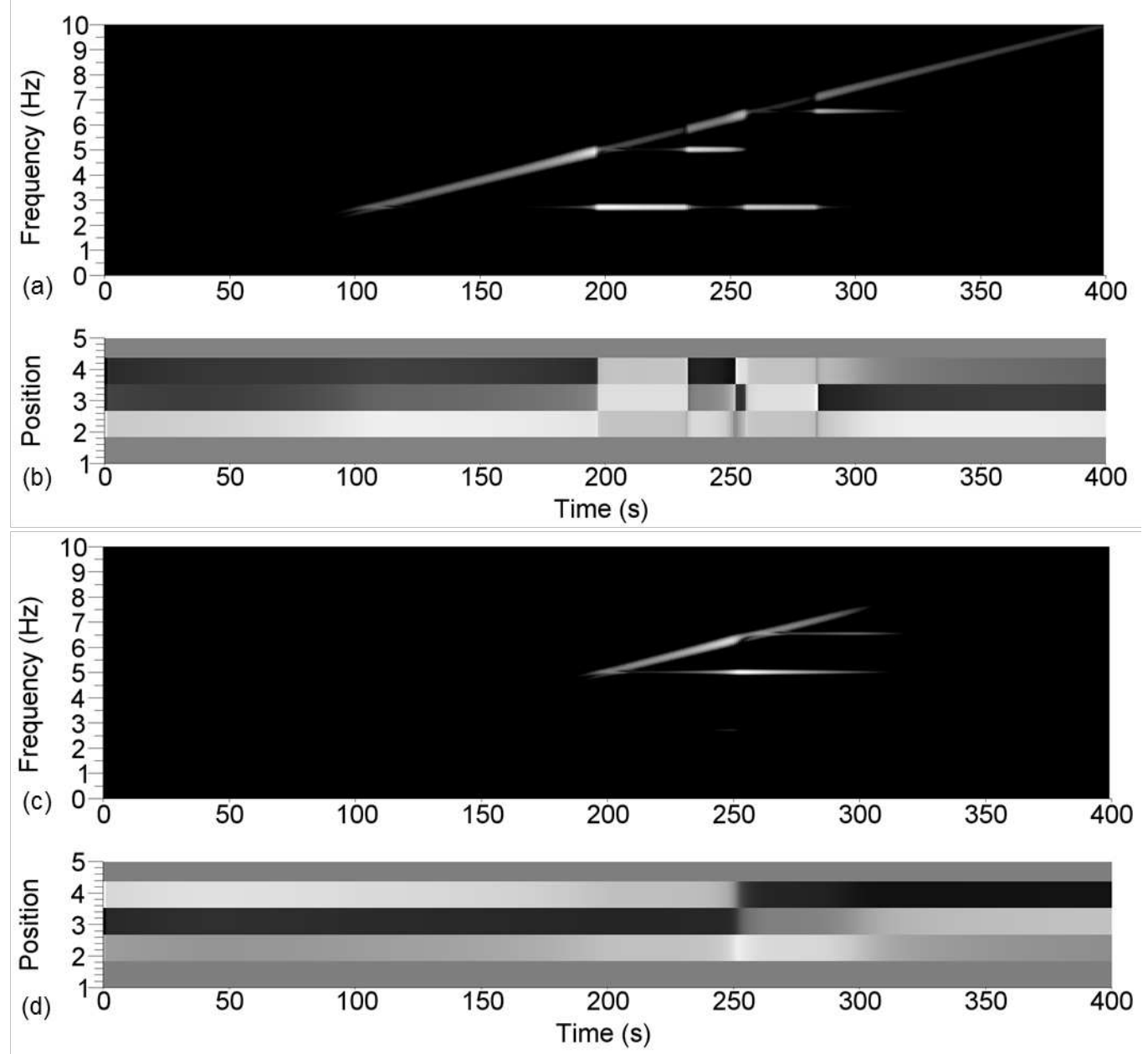

Figure 4: Results for the second and third POMs of a test with $\xi_{1}=0.1 \%$ and $F=10$ N. (a) Timefrequency representation of the second POC multiplied by the POV $\lambda_{2}^{\tau} \hat{G}_{2}(\tau, f)$. (b) Time evolution of the second POM $\Phi_{2}^{\tau}$. (c) Time-frequency representation of the third POC multiplied by the POV $\lambda_{3}^{\tau} \hat{G}_{3}(\tau, f)$. (d) Time evolution of the third POM $\Phi_{3}^{\tau}$.

\subsection{Physical interpretation of SWPOD at low excitation level}

From results discussed in [20], an analytic solution of Eq. (17) (neglecting the nonlinear terms) can be written for each modal component $q_{i}(t)$ as

$$
q_{i}(t)=\left|Q_{i}(t)\right| \sin \left(\varphi(t)-\alpha_{i}(t)\right)
$$

with $\varphi(t)$ defined by Eq. (19) and $Q_{i}(t)=\left|Q_{i}(t)\right| \mathrm{e}^{-\mathrm{j} \alpha_{i}(t)}$ a complex function (not given here). The amplitude $Q_{i}(t)$ has a maximum located a bit after the passing of the swept sine through the excitation frequency $\omega_{i}$, which characterizes the resonance of the system. After this maximum, a low-frequency oscillation of the amplitude $\left|Q_{i}(t)\right|$ occurs, which can be interpreted as the superposition of the two parts of the oscillation: free oscillations at the natural frequency, initiated during the passage of the resonance, and forced oscillations with variable excitation frequency [20]. This interpretation agrees with the results shown in Figs. 2(c) and 3(c) where the oblique frequency lines correspond to forced 
oscillations with variable excitation frequency, following the swept sine, and the horizontal frequency lines correspond to free oscillations at the natural frequencies of the system.

The energy sharing in Fig. 2(a) thus comes from the transition between the free oscillation of the first mode and the oscillation following the sweep, whereas the sharings of energy in Fig. 3(a) appear because of the simultaneous free oscillations of several modes. The sudden changes of shape present in the time-space plots Fig. 2(d), Fig. 3(d) and Fig. 4(b,d) appear at these transitions, when the energy sharing is maximum.

The test at $\xi_{1}=0.1 \%$ (Fig. 3) can then be detailed as follows. After the first resonance of the system at $\tau_{1}=112 \mathrm{~s}$, the most energetic localized POM is the first linear mode of the system during most of the time, as shown in Fig. 3(d), because of its low damping. The first POM takes the form of the second and third linear normal modes around their resonances at $\tau_{2}=203 \mathrm{~s}$ and $\tau_{3}=264$ s. These two modes decrease faster than the first one because of their higher dampings, leading to alternances between the three linear normal modes as most energetic POM. Moreover, the different slopes observed in Fig. 3(b) associated to the decay of the three linear normal modes are directly linked to the damping ratio of the modes, as shown below.

The identification of the linear normal modes of the system in term of resonance frequencies and modes shapes can be achieved through SWPOD. The local maxima of the time-energy plot of the first localized POM (which corresponds to maximum of $\left|Q_{i}(t)\right|$ ) characterize the resonance motions of the system, giving access to the resonance frequencies and mode shapes. If the local maximum occurs at $\tau_{i}$, the mode shape is then estimated by $\boldsymbol{\Phi}_{1}^{\tau_{i}}$ and the resonance frequency by $\arg \max _{f} \lambda_{1}^{\tau_{i}} \tilde{G}_{1}\left(\tau_{i}, f\right)$. Using Fig. 2 and Fig. 3, the estimation of the resonance frequencies give $2.73 \mathrm{~Hz}, 5.04 \mathrm{~Hz}$ and $6.57 \mathrm{~Hz}$. and the mode shapes $\boldsymbol{\Phi}_{1}^{\tau_{1}}=(0.70,1,0.71)^{T}, \boldsymbol{\Phi}_{1}^{\tau_{2}}=(1,0,-1)^{T}$ for both tests, with $\boldsymbol{\Phi}_{1}^{\tau_{3}}=(0.74,-1,0.69)^{T}$ when $\xi_{1}=0.5 \%$ and $\boldsymbol{\Phi}_{1}^{\tau_{3}}=(0.73,-1,0.70)^{T}$ when $\xi_{1}=0.1 \%$. These results are very close to the theoretical linear normal modes of the system, with a slight asymmetry for the third mode.

The damping ratios can also be obtained by focusing on the horizontal frequency lines. They correspond to the free oscillations at the natural frequency $\omega_{i}$, initiated during the passage of a resonance at the time $\tau_{i}$. The associated modal motion can then be approximated by :

$$
q_{i}(t)=q_{i 0} \mathrm{e}^{-\xi_{i} \omega_{i}\left(t-\tau_{i}\right)} \cos \left(\sqrt{1-\xi_{i}^{2}} \omega_{i}\left(t-\tau_{i}\right)+\alpha_{i 0}\right),
$$

where $q_{i 0}$ and $\alpha_{i 0}$ are real constants imposed when the free motion is initiated. In Eq. (22), the damping term has been commonly replaced by $\frac{c_{i}}{2 m}=2 \xi_{i} \omega_{i}$. Assuming that in the neighborhood of the passage of the resonance, the windowed vector signal is reduced to $\mathbf{S}^{\tau}(t)=\boldsymbol{\Phi}_{i} q_{i}^{\tau}(t)$ where

$$
q_{i}^{\tau}(t)=q_{i 0} \mathrm{e}^{-\frac{(t-\tau)^{2}}{2 \sigma^{2}}} \mathrm{e}^{-\xi_{i} \omega_{i}\left(t-\tau_{i}\right)} \cos \left(\sqrt{1-\xi_{i}^{2}} \omega_{i}\left(t-\tau_{i}\right)+\alpha_{i 0}\right),
$$

SWPOD method gives only one localized POM $\Psi_{1}^{\tau}=\Phi_{i}$ with the associated locatized POV

$$
\lambda_{1}^{\tau}=q_{i 0} \int_{0}^{T} \mathrm{e}^{-\frac{(t-\tau)^{2}}{\sigma^{2}}} \mathrm{e}^{-2 \xi_{i} \omega_{i} t} \cos ^{2}\left(\sqrt{1-\xi_{i}^{2}} \omega_{i} t+\alpha_{i 0}\right) \mathrm{d} t .
$$

Through a change of variables and trigonometric decompositions, Eq. (24) can be written :

$$
\lambda_{1}^{\tau}=\mathrm{e}^{-2 \xi_{i} \omega_{i} \tau}\left(c_{0}+c_{1} \cos \left(2 \sqrt{1-\xi_{i}^{2}} \omega_{i} \tau+\alpha_{i 0}\right)+c_{2} \sin \left(2 \sqrt{1-\xi_{i}^{2}} \omega_{i} \tau+\alpha_{i 0}\right)\right),
$$

where $c_{1}$ and $c_{2}$ are constant, small compared to the constant $c_{0}$. The POV $\lambda_{1}^{\tau}$ can then be considered as proportional to $\mathrm{e}^{-2 \xi_{i} \omega_{i} \tau}$ and using a logarithmic scale corresponds to a straight line with slop 
$\alpha_{i}=-2 \xi_{i} \omega_{i}$ in the time-energy plot. This property can then be used to identify the modal damping of the system.

In Fig. 2(b), the slope present after the first local maximum corresponds to the exponential decay of the first mode (see the associated horizontal frequency line Fig. 2(c)). This slope has been estimated using a linear regression giving $\alpha_{1}=-0.166$ and used to estimate the associated damping ratio, thus obtaining the value $\xi_{1}=\alpha_{1} /\left(2 \omega_{1}\right)=0.486$, which is very close to the theoretical one $\xi_{1}=0.5$. In Fig. 3(b), the slopes present after the three local maxima correspond to the exponential decay of the three modes. These slopes have been estimated respectively as $\alpha_{1}=-0.034, \alpha_{2}=-0.117$ and $\alpha_{3}=-0.199$ and used to estimate the associated damping ratios giving $\xi_{1}=\alpha_{1} /\left(2 \omega_{1}\right)=0.099 \%$, $\xi_{2}=\alpha_{2} /\left(2 \omega_{2}\right)=0.185 \%$ and $\xi_{3}=\alpha_{3} /\left(2 \omega_{3}\right)=0.241 \%$. These values are very close to the theoretical values $\xi_{1}=0.1 \%, \xi_{2}=0.185 \%$ and $\xi_{3}=0.242 \%$.

The analysis method proposed is thus able to identify the modal damping of a linear system if the sweep used is fast enough, in addition to identifying its modes and resonance frequencies.

\section{$5 \quad$ High excitation levels (nonlinear behavior)}

A moderate damping ratio $\xi_{1}=0.5 \%$ is considered in this section, with high excitation levels. The behavior of the system is expected to be nonlinear.

\subsection{Analysis of a simulated test with a nonlinear behavior}

A test was carried out at high excitation level $F=500 \mathrm{~N}$. The results obtained by the SWPOD method are reported in Fig. 5 and Fig. 6.

The proportions of energy captured by the three localized POMs are given in Fig. 5(a), showing results similar to the low amplitude case, with one dominant localized POM during most of the test. From Fig. 5(b), three resonance motions are now detected at $\tau_{1}=147 \mathrm{~s}, \tau_{2}=203 \mathrm{~s}$ and $\tau_{3}=264 \mathrm{~s}$, giving the resonance frequencies $3.66 \mathrm{~Hz}, 5.04 \mathrm{~Hz}$ and $6.57 \mathrm{~Hz}$ for which the associated localized POMs $\boldsymbol{\Phi}_{1}^{\tau_{1}}=(1,0.94,1)^{T}, \boldsymbol{\Phi}_{1}^{\tau_{2}}=(1,0,-1)^{T}$ and $\boldsymbol{\Phi}_{1}^{\tau_{3}}=(0.74,-1,0.69)^{T}$ are shown. The first resonance motion has been translated with respect to the low excitation case (see Fig. 2(b)). The associated resonance frequency is thus higher than the linear one and the associated shape now differs significantly from the first normal mode of the underlying linear system. This result is in accordance with the hardening effect of the nonlinear term. The second resonance motion is not affected by the nonlinearity, because of its position on one of the nodes of the mode. The third resonance motion seems also not affected by the nonlinearity but can be impacted by increasing the excitation amplitude.

In the next section, the resonance motions will be investigated in the framework of the nonlinear normal modes (NNM) of the associated autonomous undamped system in terms of frequency and mode shape.

The time-frequency plot (Fig. 5(c)) contains an oblique line for the sweep, as before, but on passing the first resonance motion, a curved frequency line is observed which decreases up to the first resonance frequency of the underlying linear system. As in the low excitation case (linear behavior) this curved line characterizes the free motion initiated passing the first resonance of the system. During this free motion, the decrease of the frequency is now coupled to the variation of the mode shape as shown in Fig. 5(c) for $\tau \in[147,173]$. The two limit mode shapes are shown in Fig. 5(b). The last one corresponds to the mode shape of the first mode of the underlying linear system. 


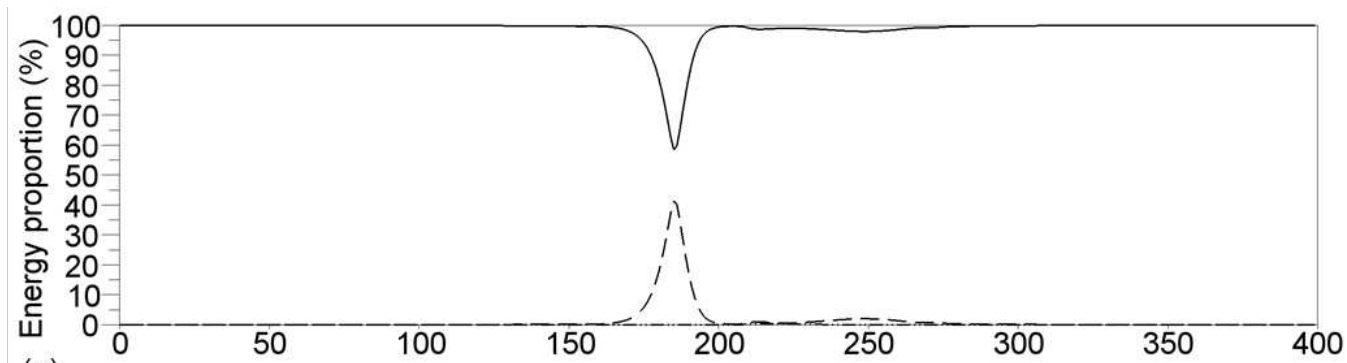

(a)
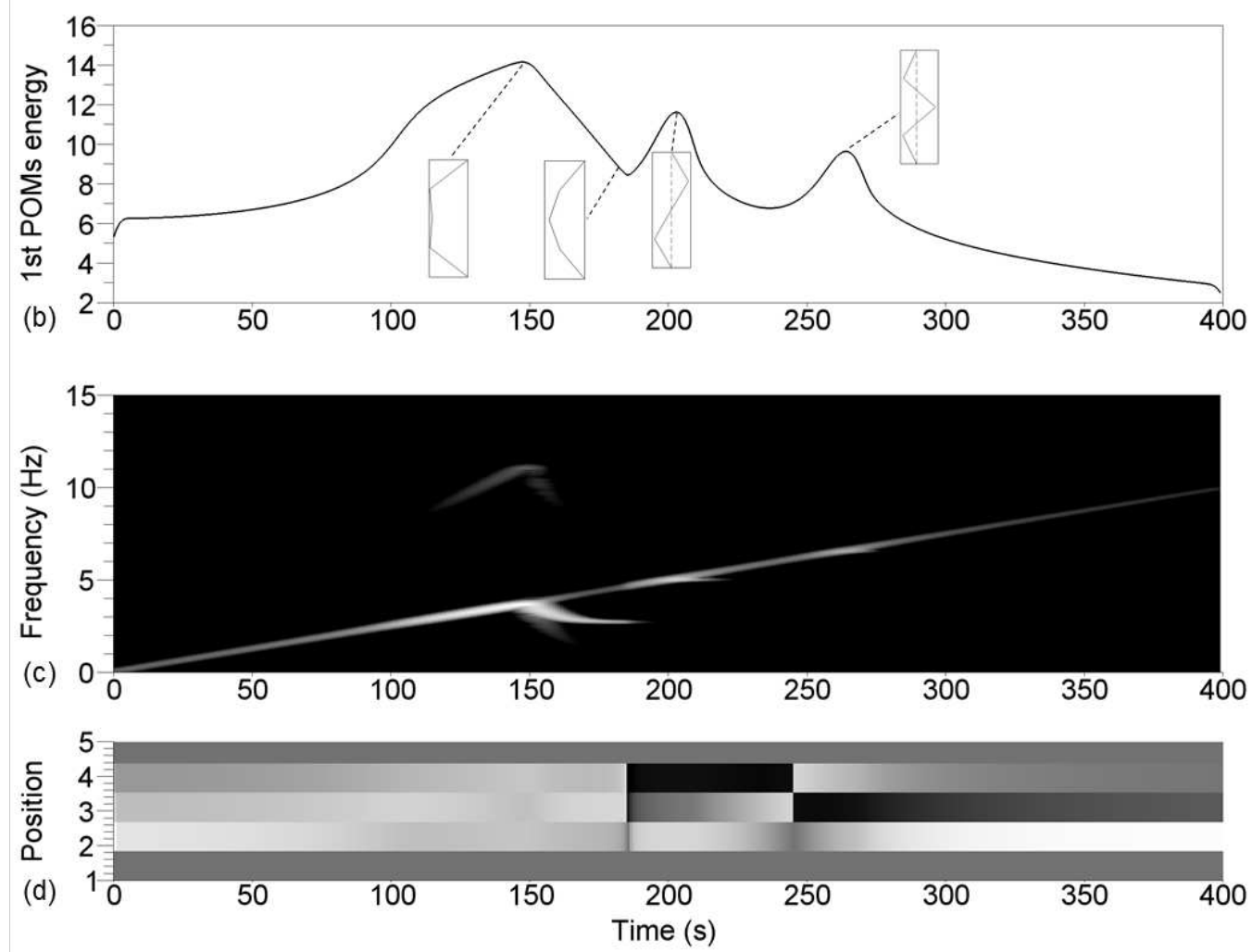

Figure 5: Results for a test with $\xi_{1}=0.5 \%$ and $F=500$ N. (a) Proportion of energy captured by the first (solid line), second (dashed) and third (dotted) POMs. (b) Energy captured by the first POM. (c) Time-frequency representation of the first POC multiplied by the POV $\lambda_{1}^{\tau} \hat{G}_{1}(\tau, f)$. (d) Time evolution of the first POM $\Phi_{1}^{\tau}$

It is interesting to note that the decay of the free response is also linear (see Fig. 5(b)). The slope value is $\alpha_{1}=-0.164$, which is the same as for the low excitation case ( $\alpha_{1}=-0.165$ in the linear case), showing that the first nonlinear mode decays with the same speed as the linear mode, not impacted by the frequency-amplitude dependence. Hence the sharing of energy between the two first localized POMs (see Fig. 5(a)) is here again a transient effect coming from the simultaneous decay of the first resonance and the vibration following the swept sine.

The time-frequency plot (Fig. 5(c)) also shows odd harmonics due to the nonlinear behavior of the system, which is mostly captured by the second POM, as shown in Fig. 6(b). 

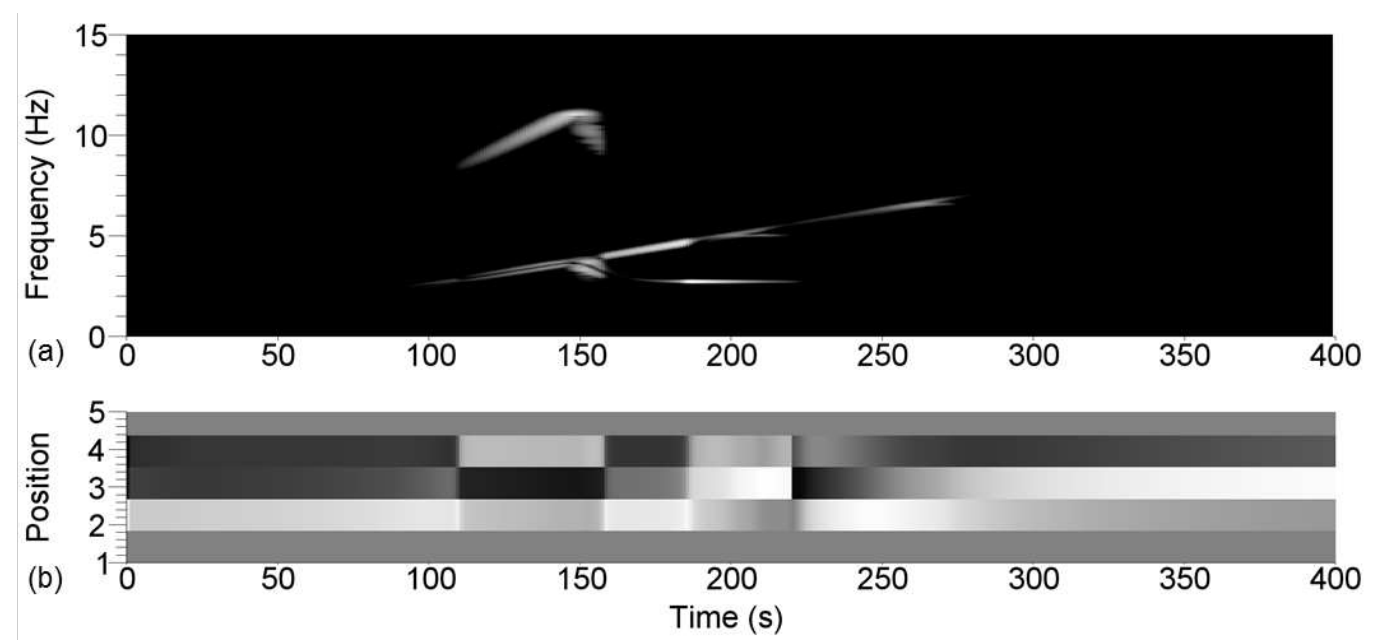

Figure 6: Results of the sliding window POD analysis for the second POMs for a test with $\xi_{1}=0.5 \%$ and $F=500 \mathrm{~N}$. (a) Time-frequency representation of the second POC $\hat{G}_{2}(\tau, f)$. (b) Time evolution of the second $\mathrm{POM} \Phi_{2}^{\tau}$.

\subsection{Physical interpretation of SWPOD at high excitation levels}

The previous analysis was repeated for 33 excitation levels $F$ from 10. N to 10000. N. For each level, the resonance motions were identifed $\left(\tau_{i}\right)$ and characterized as described in Section 4.3 in terms of frequency $\left(\arg \max _{f} \lambda_{1}^{\tau_{i}} \tilde{G}_{1}\left(\tau_{i}, f\right)\right)$ and mode shape $\left(\boldsymbol{\Phi}_{1}^{\tau_{i}}\right)$. The results are reported in Fig. 7 in a frequency-maximum displacement plot where for each component, the maximum displacement is estimated from Eq. (8) (reduced to the first term) and $\tau=\tau_{i}$. The results are compared to the NNM of the associated autonomous undamped system, defined as a familly of periodic orbits and calculated using the harmonic balance method (HBM) and the so-called Asymptotic Numerical Method (ANM) [23]. The NNMs were computed using the software ManLab [22]. With the HBM method the number of harmonics $\mathrm{H}$ has to be chosen carefully. All the numerical results were obtained using $\mathrm{H}=9$. It was checked that all the curves were only very slightly modified by increasing $\mathrm{H}$ from 9 to 11 . Only the first and third NNMs were computed, since the second NNM coincides with the second normal mode of the associated autonomous undamped system and would be represented in Fig. 7 by vertical lines at $f_{2}^{0}=5.03 \mathrm{~Hz}$ as observed with the SWPOD method.

SWPOD analysis correctly reproduces the third NNM, as well as the first NNM provided that the excitation amplitude remains relatively low. A few mode shapes obtained in this domain of validity are given in Fig. 7, with their corresponding excitation amplitudes. Considering the first NNM, differences appear indeed only at high excitation level (high displacement and frequency values, where the higher harmonic terms have an impact on the displacement of the second mass). The SWPOD, with the proposed identification method, can thus identify a NNM of a system as long as the first harmonic remains dominant i.e as long as the NNM can be considered as a similar NNM [8], i.e as long as the NNM can be captured by the first localized POM in the expansion (8). However, a way to increase the accuracy of the identification of the NNMs is under study, which combines the higher localized POMs obtained around the resonances to take into account the higher harmonics of vibration.

The proposed procedure is thus able to characterize the NNM of a nonlinear system for moderate 


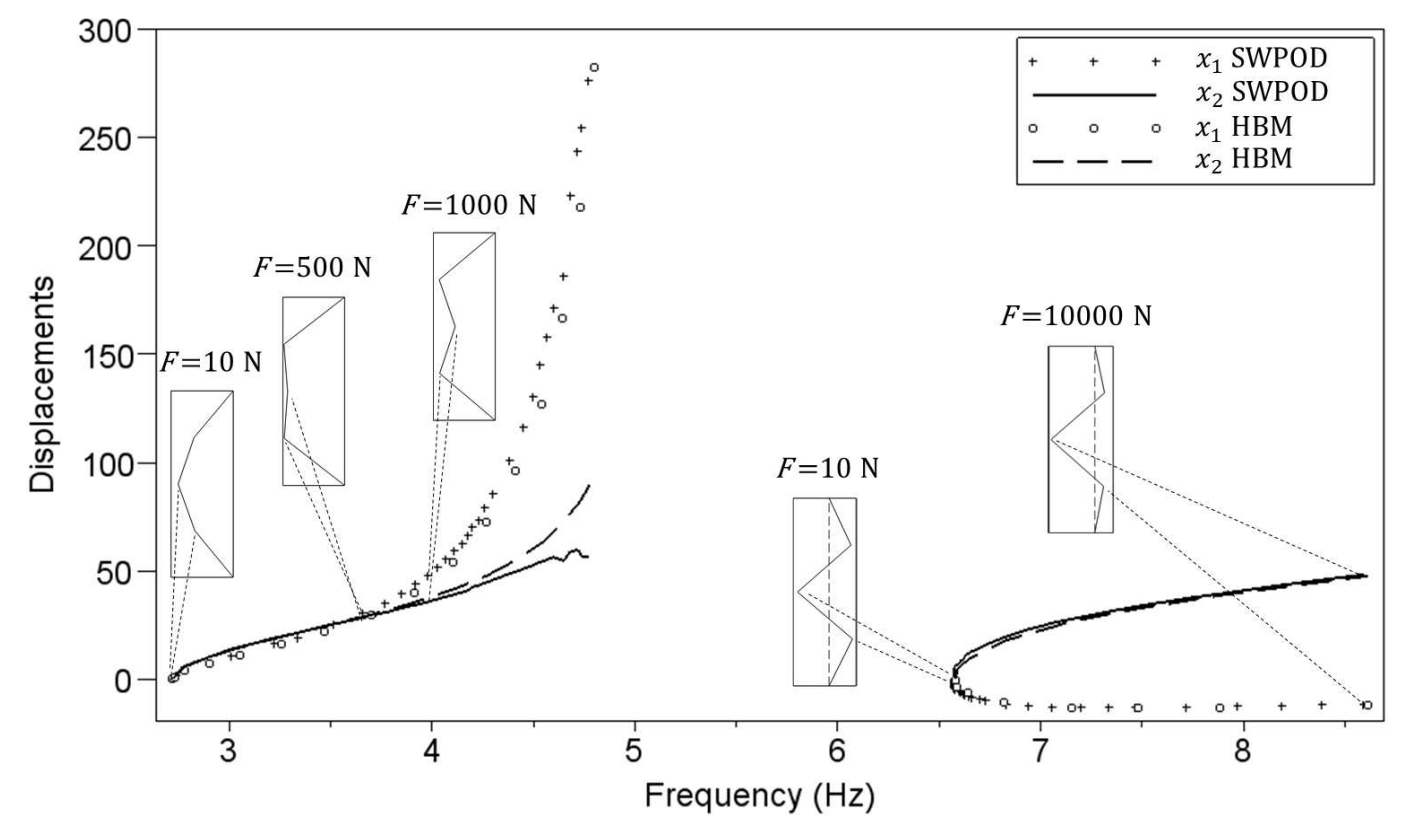

Figure 7: Frequency-maximum displacement plot: displacements of the first and second masses versus the associated resonance frequencies obtained from the simulated tests analysed with SWPOD (plus signs, full line) and the HBM (circles, dashed line) for the first (left) and third (right) NNMs. The shapes obtained at resonance are given for a few excitation amplitudes.

excitation amplitudes from response data alone.

\section{Conclusion}

An analysis method called Sliding Window POD (SWPOD), combining Proper Orthogonal Decomposition and Continuous Gabor Transform, was proposed. This method can be applied to various types of multivariate data, from experimental or simulated tests. The principal interest of the method is to give a compact representation of both structural and frequential information versus time. The theoretical formulation of the method was posed, then its practical application was detailed. The method was then applied to simulated tests of a lightly damped, nonlinear three-masses system forced following a linear swept sine. Two cases were thoroughly studied : one at low excitation level, and one at high excitation levels.

The simulated tests at low excitation level have shown that the application of the SWPOD to the simulated displacements allows the identification of the resonance frequencies, mode shapes and damping ratios of the underlying linear system. The SWPOD method also showed the ability to separate the forced vibration of the system following the swept sine and its free vibration, which appear on passing its resonances.

The simulated tests at high excitation levels have shown that the method is able to identify the nonlinear normal modes (NNM) of the system in terms of resonance frequencies and mode shapes, for moderate excitation amplitudes. The application of the method, in this paper, was focused on the analysis of structural dynamics, but various applications of the method could be done in different 
fields of research.

\section{References}

[1] Ruzzene, M., Fasana, A., Garibaldi, L., Piombo, B., Natural frequencies and damping identification using wavelet transform: Application to real data, Mechanical Systems and Signal Processing 11(2) (1997), 207-218.

[2] Argoul, P., Le, T.P., Instantaneous indicators of structural behaviour based on the continuous Cauchy wavelet transform, Mechanical Systems and Signal Processing 17 (2003), 243-250.

[3] Erlicher, S., Argoul, P., Modal identification of linear non-proportionally damped systems by wavelet transform Mechanical Systems and Signal Processing 21 (2007), 1386-1421.

[4] Le, T.P., Paultre, P. Modal identification based on continuous wavelet transform and ambient excitation tests, Journal of Sound and Vibration 331(2012), 2023-2037

[5] Lamarque, C.-H., Pernot, S., Cuer, A., Damping identification in multi-degree-of-freedom systems via a wavelet-logarithmic decrement-Part 1: Theory, Journal of Sound and Vibration 235(3) (2000), 361-374.

[6] Bellizzi, S., Guillemain P., Kronland-Martinet, R., Identification of coupled non-linear modes from free vibration using time-frequency representations, Journal of Sound and Vibration 232(2) (2001), 191-213.

[7] Camillacci, R.,Ferguson, N.S., White, P.R. Simulation and experimental validation of modal analysis for non-linear symmetric systems, Mechanical Systems and Signal Processing 19 (2005), $21-41$.

[8] Kerschen, G., Golinval, J.C., Physical interpretation of the proper orthogonal modes using the singular value decomposition, Journal of Sound and Vibration 249 (2002), 849-865.

[9] Bellizzi, S., Sampaio, R., POMs analysis of randomly vibrating systems obtained from KarhunenLove expansion, Journal of Sound and Vibration 297 (2006), 774-793.

[10] Han, S., Feeny, B., Application of proper orthogonal decomposition to structural vibration analysis, Mechanical Systems and Signal Processing 17 (2003), 989-1001.

[11] Ricciardi, G., Bellizzi, S., Collard, B., Cochelin, B., Row of fuel assemblies analysis under seismic loading: Modelling and experimental validation, Nuclear Engineering and Design 239, Issue 12 (2009), 2692-2704.

[12] Kerschen, G., Golinval, J.C., Feeny, B., Vakakis, A.F., Bergman, L.A., The Method of Proper Orthogonal Decomposition for Dynamical Characterization and Order Reduction of Mechanical Systems : An Overview, Nonlinear Dynamics 41 (2005), 147-169.

[13] Hassanpour, H., A time-frequency approach for noise reduction, Digital Signal Processing 18 (2008), 728-738. 
[14] Kutz, J. N., Data-Driven Modeling $\&$ Scientific Computation. Methods for Complex Systems $\&$ Big Data, Oxford, New York, 2013.

[15] Yin, H.P, Duhamel, D., Argoul, P., Natural frequencies and dampings estimation using wavelet transform of a frequency response function, Journal of Sound and Vibration 271 (2004), 999-1014.

[16] Zhang, Z.Y., Hua, H.X., Xu, X.Z, Huang, Z., Modal parameter identification through Gabor expansion of response signals, Journal of Sound and Vibration 266 (2003), 943-955.

[17] Ricciardi, G., Boccaccio, E., Stiffening of a fuel assembly under axial flow, Proccedings of the 9th International Conference on Flow-Induced Vibration (\& Flow-Induced Noise)- FIV2012, Dublin, Ireland, (2012), 391-398.

[18] Feeny, B.F., A Complex Orthogonal Decomposition for Wave Motion Analysis, Journal of Sound and Vibration 310(1) (2008), 77-90.

[19] Gloth, G., Sinapius, M., Analysis of swept-sine runs during modal identification, Mechanical Systems and Signal Processing 18 (2004), 1421-1441.

[20] Markert, R., Seidler, M., Analytically based estimation of the maximum amplitude during passage through resonance, International Journal of Solids and Structures 38 (2001), 1975-1992.

[21] Gabor, D., Theory of communication, Journal of I.E.E.E. (1946), 93,429-441.

[22] ManLab, 2010. Version 2.0. (http://manlab.lma.cnrsmrs. fr/).

[23] Cochelin, B., Vergez, C., A high order purely frequency-based harmonic balance formulation for continuation of periodic solutions, Journal of Sound and Vibration, 324 (2009), 243-262. 